\title{
INVESTIGATING POTENTIAL SOURCES OF TOXOPLASMOSIS FOR HUMANS FROM SLAUGHTERED FOOD ANIMALS IN IBADAN, NIGERIA
}

\author{
Ayinmode, A. B. ${ }^{1}$, Abiola, J. O. ${ }^{2}$ \\ ${ }^{1}$ Department of Veterinary Microbiology and Parasitology \\ ${ }^{2}$ Large animal unit, Department of Veterinary Medicine, Faculty of Veterinary Medicine \\ University of Ibadan, Nigeria \\ ayins2000@yahoo.com
}

\section{ABSTRACT}

The consumption of undercooked meat by humans is a potential infectious source for Toxoplasmosis. This study was aimed at finding potential infectious sources of Toxoplasma gondii for humans by investigating the seroprevalence of $T$. gondii in animals slaughtered in the Ibadan municipal abattoir. Serum samples from 1337 slaughtered animals (477 cattle, 267 sheep, 139 goats, and 454 pigs) were analyzed for the presence of antibodies to Toxoplasma gondii. Serological studies using the ELISA method demonstrated the prevalence of T. gon-

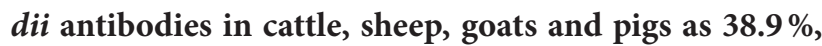
$1.9 \%, 3.6 \%$ and $45.2 \%$, respectively. Univariate statistical analysis detected an association between $T$. gondii seropositivity and sheep, goat and $\operatorname{sex}(\mathrm{P}<0.05)$. In the multivariate logistic regression model, only sheep, goats and pigs had an association with $T$. gondii seropositivity, while sex was a confounding factor. The detection of varying levels of antibodies to $T$. gondii infection in these food animals highlights their potential as a source of T. gondii for humans. Efforts should, therefore be di- rected at preventing the infection during the production and the processing of meat for food.

Key words: cattle; goats; infection; Nigeria; pigs; slaughter; Toxoplasma gondii

\section{INTRODUCTION}

Toxoplasmosis is one of the most common parasitic zoonosis caused by Toxoplasma gondii, an intracellular apicomplexan protozoan that infects almost all warm-blooded animals, including domestic animals and humans [9], [11].

T. gondii infection in humans is influenced by the immune status and genetic composition of the host and the timing of the infection [13]. Acquired T. gondii infections are usually asymptomatic, although lymphadenopathy or ocular disease may occur in some patients [5]. T. gondii is also an important cause of abortions and stillbirths in infected pregnant women and has been associated with fetal anomalies [25]. Congenital toxoplasmosis may cause retinal and mental retardation, seizures, blindness, and death [17]. 
Some cases of acute toxoplasmosis have been associated with psychiatric symptoms such as delusions and hallucinations [17] and psychiatric manifestations have been observed in immunocompromised persons with AIDS in whom latent infections with $T$. gondii have been reactivated [16].

T. gondii infects humans through the ingestion of food or water contaminated with oocysts shed by cats (the definitive host) or by eating undercooked or raw meat containing tissue cysts, especially the viscera of the intermediate hosts $[13,8]$. Several studies worldwide have identified undercooked meat (lamb, beef or game) as a major risk factor for toxoplasmosis in humans [11], [19]. In addition, the presence of cats, feed source, farm management, methods of rodent and bird control, carcass handling and water quality have also been suggested as risk factors for the infection in livestock [14], [31].

Seroprevalence studies worldwide showed that $T$. gondii infections are widespread in food animals with results varying between and within regions. Furthermore, the outcome of tests depends on sampled species and/or breed, type of test, the cut-off values and most times the sample size of the study [11]. The seroprevalence of T. gondii has been found to be higher in pigs, sheep and poultry than in cattle and less in meat product of animals raised indoor than outdoor reared animals exposed to food, water and environment contaminated with oocyst [14].

The seroprevalence of $T$. gondii antibodies in livestock may be an indirect method to predict potential infection sources, since a correlation was determined between antibodies to T. gondii and the presence of tissue cysts in sheep and pigs [26]. However, this may not be the case with cattle where tissue cyst are rarely found even when antibodies were detected in up to $92 \%$ of the cattle examined [30]. Tissue cysts of $T$. gondii are often detected in the tissues of sheep, goats and pigs compared with other domestic animals [30].

The seroprevalence of $T$. gondii antibodies obtained in human populations has also been found to vary greatly between countries, among different geographical areas within same country and among different ethnic groups living in the same area [30]. In Nigeria, there are several published seroprevalence studies indicating T. gondii infection is endemic among both immunocompromised and immunocompetent individuals [27], [28]. Nevertheless, the sources of T. gondii infection in humans has not been reported. A study by Ka mani et al. [18] on the risk factors for T. gondii infection among cat and dog owners in Northern Ni- geria, suggested that contact with pets are not likely to be the main source of infections for humans. While, ingestion of contaminated meat might be a potential source of transmission of T. gondii to humans in Nigeria, no report is available to verify it as an infection source. This study was therefore aimed at investigating whether animals slaughtered for food in the Ibadan municipal abattoir could be a potential source of T. gondii infections for humans.

\section{MATERIALS AND METHODS}

\section{Location and sampling}

The sampling site for the study was the Bodija abattoir in Ibadan, Oyo state, Nigeria. Located at $7^{\circ} 23^{\prime} 47^{\prime \prime} \mathrm{N}$, $3^{\circ} 55^{\prime} 0^{\prime \prime} \mathrm{E}$, Ibadan is one of the largest cities in West Africa with a land area of $3,080 \mathrm{~km}^{2}$ and a population of 2,338,659 [5]. The Bodija abattoir is the largest slaughter facility available in Ibadan and accounts for $65.93 \%$ of the total animals slaughtered in Oyo state [1]. Blood samples were randomly collected from 1337 animals (267 sheep, 139 goats, 477 cattle, and 454 pigs) at slaughter over a period of one year. The samples were collected into sterile tubes without anticoagulant and left at room temperature $\left(29-33^{\circ} \mathrm{C}\right)$ for $1-2$ hours. The separated sera were harvested and stored at $-20^{\circ} \mathrm{C}$ until used for serology.

\section{Serology}

The serum samples obtained were analyzed using the commercially available ID Screen Toxoplasmosis Indirect Multi-Species ELISA Kits (ID Vet Innovative Diagnostic, Montpellier, France) to determine the presence of T. gondii specific IgG antibodies. The procedure for the test and interpretation of the results obtained were performed according to the manufacturer's instructions.

\section{Statistical Analysis}

The data were first entered and stored in Microsoft excel spreadsheet and afterwards exported into SPSS 20 statistical software (SPSS Inc., Chicago, IL, USA). The univariate analysis was done to evaluate the strength of the relationship between our outcome variable and the explanatory variables. The independent effects of variables associated with our dependent variable at $\mathrm{P}<0.05$ on univariate analysis were entered into the multivariate logistic regression model to further examine the predictors' effect on our response vari- 
able. Similarly, Negelkerke's R2 was used in our analysis to illuminate how much variance in the dependent variable is explained or affected by predictor variables. The data were therefore analyzed based on the $5 \%$ level of significance.

\section{RESULTS}

Table 1 gives a summary of the characteristics of the sampled animals at the Ibadan municipal abattoir. Table 2 highlights the distribution of $T$. gondii infection across species, age and sex of animals slaughtered at the Ibadan

Table 1. Physical characteristics of animals slaughtered at the Ibadan municipal abattoir

\begin{tabular}{|c|c|c|}
\hline Variable & $\mathbf{N}$ & $\%$ \\
\hline \multicolumn{3}{|c|}{ Age } \\
\hline Young & 25 & 1.9 \\
\hline Grower & 305 & 22.8 \\
\hline Adult & 1007 & 75.3 \\
\hline & Sex & \\
\hline Male & 660 & 49.3 \\
\hline Female & 677 & 50.6 \\
\hline \multicolumn{3}{|c|}{ Pig } \\
\hline Large white & 288 & 63.3 \\
\hline Durock & 96 & 21.1 \\
\hline Black local & 47 & 10.3 \\
\hline Crossed(LW+D) & 23 & 5.1 \\
\hline \multicolumn{3}{|c|}{ Sheep } \\
\hline Yankasa & 30 & 11.2 \\
\hline Uda & 237 & 88.8 \\
\hline \multicolumn{3}{|c|}{ Goat } \\
\hline Sokoto red & 139 & 100 \\
\hline \multicolumn{3}{|c|}{ Cattle } \\
\hline Kuri & 34 & 7.1 \\
\hline Ndama & 10 & 2.1 \\
\hline Red Bororo & 152 & 31.7 \\
\hline Sokoto gudali & 86 & 18.0 \\
\hline White Fulani & 170 & 35.6 \\
\hline Mixed breed & 25 & 5.0 \\
\hline
\end{tabular}

Table 2. Distribution of Toxoplasma gondii infection across species, age and sex of animals slaughtered the Ibadan municipal abattoir

\begin{tabular}{|c|c|c|c|}
\hline Variable & $\mathrm{N}$ & $\begin{array}{c}\text { Positive } \\
\text { [\%] }\end{array}$ & $\begin{array}{c}\text { Negative } \\
{[\%]}\end{array}$ \\
\hline \multicolumn{4}{|c|}{ Species } \\
\hline Swine & 454 & $205(45.2)$ & $249(54.8)$ \\
\hline Ovine & 267 & $5(1.9)$ & $262(98.1)$ \\
\hline Caprine & 139 & $5(3.6)$ & $134(96.4$ \\
\hline Cattle & 477 & 186 (38.9) & $291(61.0)$ \\
\hline \multicolumn{4}{|c|}{ Age } \\
\hline Young & 25 & $10(40.0)$ & $15(60.0)$ \\
\hline Grower & 305 & $103(33.8)$ & $202(66.2)$ \\
\hline Adult & 1007 & $287(28.5)$ & 720 (71.5) \\
\hline \multicolumn{4}{|c|}{ Sex } \\
\hline Male & 660 & $150(22.7)$ & $510(77.3)$ \\
\hline Female & 677 & 248 (36.6) & $430(63.4)$ \\
\hline Total & 1337 & $\begin{array}{c}401 \\
(29.9]\end{array}$ & $\begin{array}{c}936 \\
(70 \%)\end{array}$ \\
\hline
\end{tabular}

municipal abattoir. The analysis of 1337 serum samples obtained from animals slaughtered at the municipal abattoir in Ibadan showed that 401 (29.9\%) samples were positive for T. gondii antibodies. Based on species, the prevalence of $T$. gondii antibodies were $1.9 \%$ (5/267), 3.6\% (5/139), $38.9 \%(186 / 477)$ and $45.2 \%(205 / 454)$ for sheep, goats, cattle and pigs respectively. Fig. 1 shows the distribution of T. gondii infection across various breeds of animals slaughtered at the abattoir. Of the 267 sheep examined, only five (1.9\%) samples were positive. T. gondii antibodies were detected in five $(3.6 \%)$ of the 139 goats sampled. A total of $186(38.9 \%)$ were positive out of the 477 cattle serum analyzed, while 205 (45.2\%) were positive for T. gondii antibodies of the 454 pigs sampled. There were 287 (28.5\%) adult animals positive for T. gondii antibodies while 248 $(36.6 \%)$ were females out of the total of 1337 samples analyzed from the abattoir.

On univariate analysis, T. gondii infection was significantly associated with ovine and caprine species, and also the male gender. Multivariate logistic regression model obtained a significant association between T. gondii infection and porcine, caprine and ovine species, but sex was identified as a confounder and not a factor in T. gondii infection (Table 3). 
Table 3. Multivariate logistic regression analysis of independent variables associated with Toxoplasma infection in animals slaughtered at Ibadan municipal abattoir

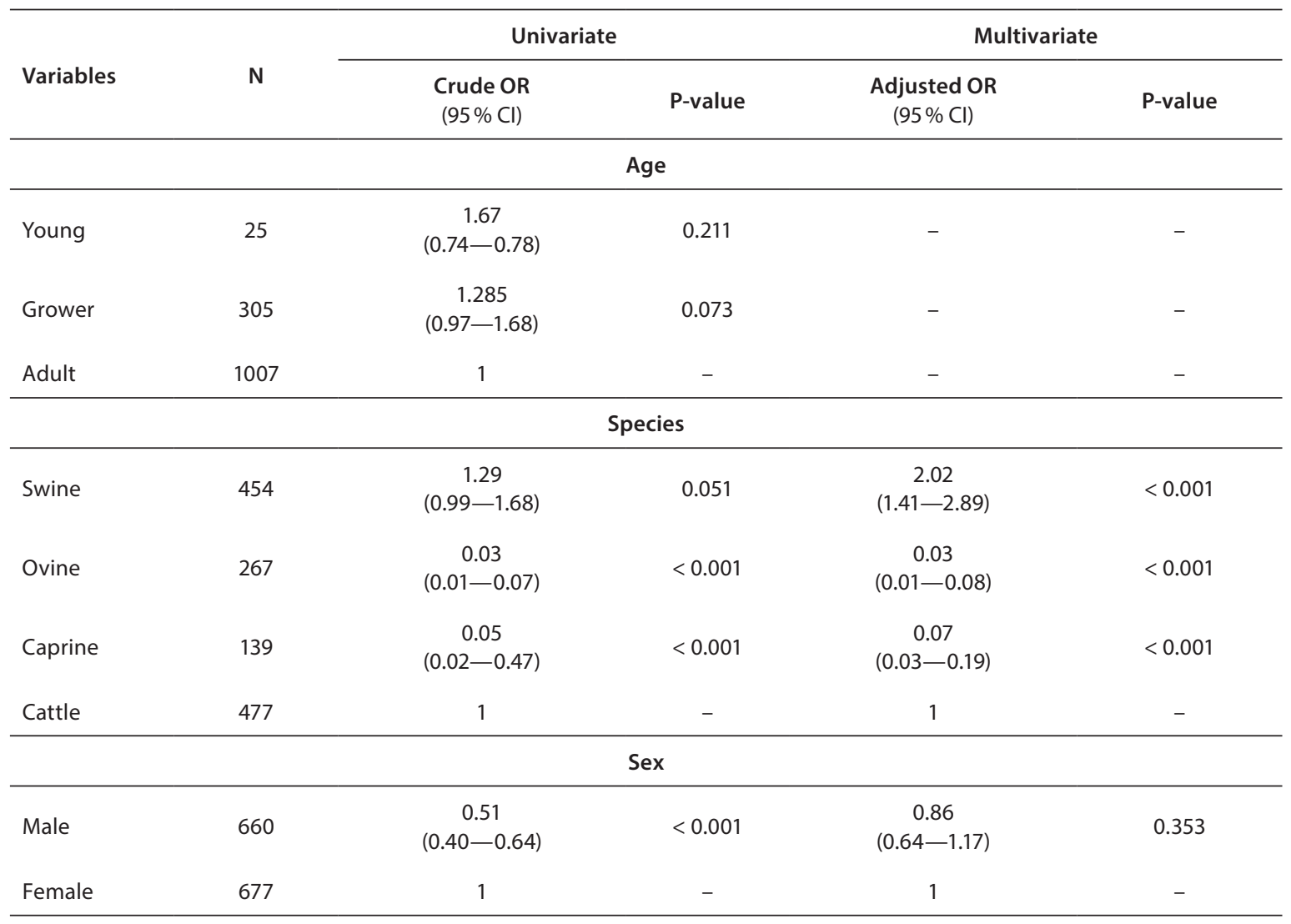

Negelkerke's R2 = 0.274; OR — Odds Ratio; $\mathrm{Cl}$ - Confidence Interval

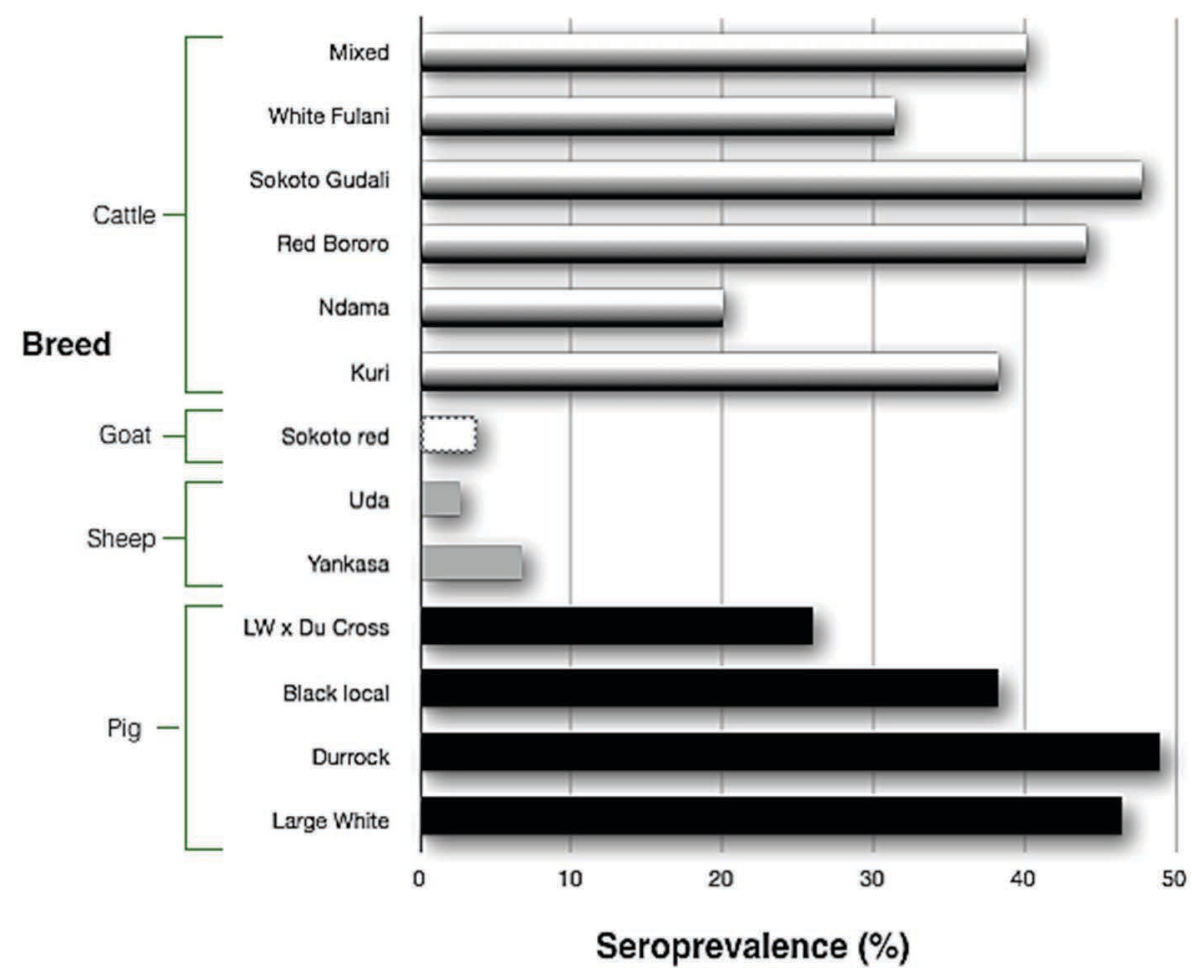

Fig. 1. Chart showing the distribution of $T$. gondii infection across various breeds slaughtered at the Ibadan municipal abattoir 


\section{DISCUSSION}

This study detected antibodies to T. gondii in all of the species of animals (cattle, sheep, pigs and goats) slaughtered at the abattoir. This indicates that T. gondii is wide spread across the region and livestock (irrespective of their species) are constantly exposed to T. gondii oocyst in their environment.

The comparison of the seroprevalence of T. gondii antibodies in our study with other reports elsewhere, show that our findings in pigs is comparable to $30.6 \%$ in Chongqing [32], 46\% in Ústí nad Labem (Czech Republic) and lower than 76\% in Hradec Králové (Czech Republic) [4]. However, our seroprevalence figure is higher than $2.5 \%$ in Finland [15], 5\% in Sweden [22], 9.8\% in North Portugal [20], 11.7\% in Nepal [7] and 13\% in Mexico [3]. For cattle, the seroprevalence detected in our study was higher than $29.1 \%$ in southern Brazil [23], 7.5\% in North Portugal [20], 6.0\% in Northeast China [21], but lower than $46.4 \%$ in Xinjiang, China [24]. For sheep, we obtained a seroprevalence lower than $33.6 \%$ in North Portugal [20], $20 \%$ in Central Ethiopia [13] and 35\% in Iran [29] and 36.4\% in Saudi Arabia [2]. For goats, we detected a seroprevalence far less than $15.5 \%, 18.5 \%, 30 \%$ and $35.3 \%$ obtained in Central Ethiopia [13], North Portugal [20], Iran [29] and Saudi Arabia [2].

The seroprevalence of T. gondii in food animals varies between and within countries across the world. Several factors including, management systems, climate, population of cat and variation (in sensitivity and specificity) of detection methods usually influence the prevalence of T. gondii reported in animals processed for meat [11]. Hence, it is therefore difficult to draw conclusive inferences when comparing T. gondii data from several different locations. However, the present findings along with reports from previous investigators suggest that humans consuming animals slaughtered around the world may be potentially exposed to $T$. gondii infection and therefore the disease should be considered in meat inspection procedures worldwide.

We obtained high seroprevalence of T. gondii antibodies in pigs brought for slaughter; a situation similar to the reports of previous investigators [32], [4]. Besides, T. gondii seropositivity was significantly associated with pigs after a multivariate logistic regression analysis. Although the reason for this observation is not known. However, the management systems under which the pigs were raised may be an important factor that influenced the outcome of our findings. Pigs in the study area are usually reared in semi-intensive and backyard systems with poor housing facilities that can easily be accessed by rodents and cats. Our previous findings on pig farms in Nigeria (unpublished data) showed that, unlike in the developed countries, domestic and feral cats regularly besiege piggeries and animal feed mills to kill rodents, thereby increasing the probability of pigs contacting viable oocyst of $T$. gondii from cats.

Reports from Europe and the USA showed that organic pigs raised with free access to outdoor environment have higher antibodies titre to T. gondii. Furthermore, pork has generally been considered as a major source of $T$. gondii infection in humans [30]. The consumption of contaminated pork was also linked to clinical toxoplasmosis in Korea [6]. With available reports on the evidence of high seroprevalence of $T$. gondii in HIV-infected individuals as well as immunocompetent humans in Nigeria [27], [28], coupled with an increase in the consumption of pork as a cheap source of protein (due to the rising cost of beef, goat meat and chickens); the detection of high levels of antibodies to T. gondii in pigs slaughtered for food, raises concern about the possible role of pork in the transmission of T. gondii to humans in Nigeria. Moreover, it has been suggested that the probability of isolating viable $T$. gondii parasites increases with high antibody titre in pigs [10]. This implies that high levels of antibodies to T. gondii in commercial pigs slaughtered for human consumption could increase the possibility of consumption of viable cyst if the pork is not properly processed. Thus, more studies are needed to investigate the role of pork consumption in the epidemiology of toxoplasmosis.

Our study observed very low antibody levels to T. gondii in small ruminants compared to the results from most other investigators [2], [13], [20]. This could be because of the varying types of management systems in our study area. Sheep and goats raised for food in Southwestern Nigeria are mostly reared in confinement with little access to the outdoor environment. This contrast with sheep and goat production from other free-ranging systems where animals are under increased pressure of T. gondii infection from oocysts in the contaminated environment [30].

Our study detected a higher prevalence of T. gondii antibodies in cattle than in sheep and goat. However, unlike with small ruminants both univariable and multivariable logistic regression analysis showed no significant associa- 
tion between T. gondii infection and cattle. Previous investigators have suggested that T. gondii infection may be of less importance in cattle than in small ruminants because the parasites are rarely isolated from naturally infected cattle [20]. Furthermore, there is evidence to show that tissue cysts are rarely found even in cattle with a seroprevalence as high as $92 \%$ [30]. It has also been suggested that cattle might have the ability to eradicate the T. gondii from their tissues [12]; hence, unlike in small ruminants, there is no correlation between antibodies to T. gondii and the presence of tissue cysts in cattle [26].

Our study found varying level of seroprevalence among age groups, breeds and the sex of food animals, but age and breed were not significantly associated with seropositivity with T. gondii. These findings contrasts with some reports that identified age as a risk factor for the infection in some livestock [13], [20]. Our study did not find sex as an independent predictor of seropositivity to $T$. gondii, but a confounding factor (in the multivariable logistic model). It is worth noting that limited inferences can be drawn on risk factors from an abattoir-based study, since we have no information on the management system of animals, presence of cats and rodents on the farm, food and water sources for animals brought for slaughter. Hence, more studies are needed for tracking T. gondii infection in livestock meant for human food from farms to slaughter. In addition, there is a need for enforcement of procedures that will ensure that detailed data on livestock brought to the abattoir are obtained before they are slaughtered.

\section{CONCLUSIONS}

This study showed that cattle, sheep, goats and pigs slaughtered for human consumption in the studied area had previous varying level of exposure to $T$. gondii infection and may serve as potential infectious sources for T. gondii infections in humans, if meat product from them are not properly handled or processed. While, the presence of high antibodies in slaughtered livestock does not directly provide hazards to humans, because the meat may not contain viable T. gondii cyst, it is imperative that the risk of zoonosis from infected food animals are not discounted. Preventive measures, especially educating the public (meat handlers and consumers) on zoonotic implication of T. gondii is highly recommended.

\section{REFERENCES}

1. Abiola, S.S., 1995: Assessment of abattoir and slaughter slab operation in Oyo State. Nig. J. Ani. Prod., 5, 54- 62.

2. Alanazi, A.D., 2013: Determination of seropositivity for Toxoplasma gondii in sheep, goats and camels slaughtered for food and human consumptions in Riyadh municipal abattoirs, Saudi Arabia. J. Egypt. Soc. Parasitol., 43, 569-76.

3. Alvarado-Esquivel, C., Vazquez-Morales, R. F., ColadoRomero, E.E., Guzmán-Sánchez, R., Liesenfeld, O., Dubey, J.P., 2015: Prevalence of infection with Toxoplasma gondii in landrace and mixed breed pigs slaughtered in Baja California sur state, Mexico. Eur. J. Microbiol. Immunol., (Bp), 5, 112-5.

4. Bártová, E., Sedlák, K., 2011: Seroprevalence of Toxoplasma gondii and Neospora caninum in slaughtered pigs in the Czech Republic. Parasitol., 138, 1369-1371.

5. Census 2006: Legal Notice on Publication of the Details of the Breakdown of the National and State Provisional Totals 2006 Census. Federal Republic of Nigeria Official Gazette (15th May 2007).

6. Choi, W.Y., Nam, H.W., Kwak, N.H., Huh, W., et al., 1997: Foodborne outbreaks of human toxoplasmosis. J. Infect. Dis., 175, 1280-1282.

7. Devleesschauwer, B., Pruvot. M., Joshi, D. D., De Craeye, S., et al., 2013: Seroprevalence of zoonotic parasites in pigs slaughtered in the Kathmandu Valley of Nepal. Vector Borne Zoonotic Diseases, 13, 872-876.

8. Dubey, J. P., 2004: Toxoplasmosis - a waterborne zoonosis. Vet. Parasitol., 126, 57- 72.

9. Dubey, J. P., 2009: History of the discovery of the life cycle of Toxoplasma gondii. International J. Parasitol., 39, 877-882.

10. Dubey, J. P., 1994: Toxoplasmosis. J. Am. Vet. Med. Assoc., 205, 1593-1598.

11. Dubey, J. P., 2010: Toxoplasmosis of Animals and Humans, 2nd edn., CRC Press, Boca Raton, 313 pp.

12. Dubey, J.P., Thulliez, P., 1993: Persistence of tissue cysts in edible tissues of cattle fed Toxoplasma gondii oocysts. Am. J. Vet. Res., 54, 270-273.

13. Gebremedhin, E.Z., Abdurahaman, M., Hadush. T., Tessema, T.S., 2014: Seroprevalence and risk factors of Toxoplasma gondii infection in sheep and goats slaughtered for human consumption in Central Ethiopia. BMC Res. Notes, 7, 696.

14. Guo, M., Dubey, J.P., Hill, D., Buchanan, R.L., et al., 2015: Prevalence and risk factors for Toxoplasma gondii infection in meat animals and meat products destined for human consumption. J. Food. Prot., 78, 457-76. 
15. Hirvela-koski, V., 1992: The prevalence of toxoplasma antibodies in swine sera in Finland. Acta Vet Scand., 33, 21-25.

16. Israelski, D. M., Remington, J. S., 1988: Toxoplasmic encephalitis in patients with AIDS. Infect. Dis. Clin. North Am., 2, 429-445.

17. Jones, J.L., Lopez, A., Wilson, M., Schulkin, J., Gibbs, R., 2001: Congenital toxoplasmosis: a review. Obstet. Gynecol. Surv., 56, 296-305.

18. Kamani, J., Mani, A.U., Egwu, G.O., Kumshe, H.A., 2009: Seroprevalence of human infection with Toxoplasma gondii and the risk factors, in Maiduguri, Borno state, Nigeria. Ann. Trop. Med. Parasitol., 103, 317-321.

19. Kijlstra, A., Jongert, E., 2008: Control of the risk of human toxoplasmosis transmitted by meat. Int. J. Parasitol., 38, $1359-1370$.

20. Lopes, A. P., Dubey, J.P., Neto, F., Rodrigues, A., et al., 2013: Seroprevalence of Toxoplasma gondii infection in cattle, sheep, goats and pigs from the North of Portugal for human consumption. Vet. Parasitol., 193, 266-269.

21. Liu, X., Liu, C., Liu, Y., Jin, H., et al., 2012: Seroprevalence of Toxoplasma gondii infection in slaughtered pigs and cattle in Liaoning province, northeastern China. J. Parasitol., 98, $440-441$.

22. Lunden, A., Lind, P., Engvall, E. O., Gustavsson, K., Uggla, A., Vagsholm, I., 2002: Serological survey of Toxoplasma gondii infection in pigs slaughtered in Sweden. Scand. J. Infect. Dis., 34, 362-365.

23. Macedo, M.F.S.B., Macedo, C.A.B., Barros, L.D., Martins, G. F., et al., 2012: Serum occurrence of anti-Toxoplasma gondii antibodies in dairy cows slaughtered in an abattoir for human consume. Cienc. Rural, 42, 1065-1069.
24. Mi, X. Y., Ba, Y.C. H., Li, W.C., 2007: Epidemic investigation of Toxoplasma gondii infection in pigs, cattle and sheep in Xinjiang. Chinese J. Vet. Parasitol., 15, 22-24.

25. Montoya, J.G., Liesenfeld, O., 2004: Toxoplasmosis. Lancet, 363, 1965-1976.

26. Opsteegh, M., Swart, A., Fonville, M., Dekkers, L., van der Giessen, J., 2011: Age-related Toxoplasma gondii seroprevalence in Dutch wild boar inconsistent with lifelong persistence of antibodies. Plos One, 6, 16240.

27. Ogoina, D., Onyemelukwe, G. C., Musa, B. O., Obiako, R O., 2013: Seroprevalence of IgM and IgG antibodies to Toxoplasma infection in healthy and HIV-positive adults from Northern Nigeria. J. Infect. Dev. Ctries., 13, 398-403.

28. Osunkalu, V. O., Akanmu S. A., Ofomah, N. J., Onyiaorah, et al., 2011: Seroprevalence of Toxoplasma gondii IgG antibody in HIV-infected patients at the Lagos University Teaching Hospital. HIV AIDS (Auckl.), 3, 101-105.

29. Sharif, M., Gholami, S. H., Ziaei, H., Daryani, A., et al., 2007: Seroprevalence of Toxoplasma gondii in cattle, sheep and goats slaughtered for food in Mazandaran province, Iran, during 2005. Vet. J., 174, 422-424.

30. Tenter, A.M., Heckeroth, A.R., Weiss, L.M., 2000: Toxoplasma gondii: from animals to humans. International. J. Parasitol., 30, 1217-258.

31. Tzanidakis, N., Maksimov, P., Conraths, F. J., Kiossis, E., et al., 2012: Toxoplasma gondii in sheep and goats: Seroprevalence and potential risk factors under dairy husbandry practices. Vet. Parasitol., 190, 340-348.

32. Wu, D., LV, R., Sun, X., Shu, F., et al., 2012: Seroprevalence of Toxoplasma gondii antibodies from slaughter pigs in Chongqing, China. Trop. Anim. Health Prod., 44, 685-687. 